\title{
Synthesis and Cytoxicity of Sempervirine and Analogues
}

Xiaohong Pan ${ }^{\mathrm{a}}$, Chunying Yang ${ }^{\mathrm{b}}$, John L. Cleveland ${ }^{\mathrm{b}}$, and Thomas D. Bannister $^{a *}$

${ }^{a}$ The Scripps Research Institute, Department of Chemistry, Jupiter, Florida 33458, USA;

${ }^{b}$ Moffitt Cancer Center \& Research Institute, Department of Tumor Biology, Tampa, Florida 33612, USA

\section{Supporting Information}

${ }^{1} \mathrm{H}$ and ${ }^{13} \mathrm{C}$ NMR Spectra for new compounds 


\section{${ }^{1} \mathrm{H}$ and ${ }^{13} \mathrm{C}$ NMR spectrum of 1 (DMSO)}

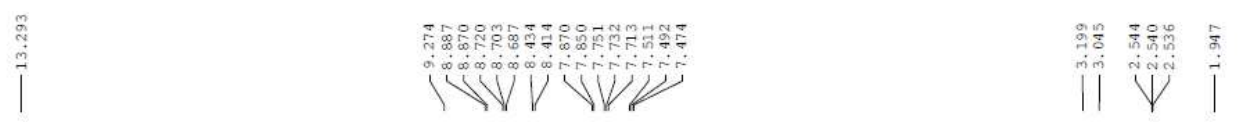

${ }^{1} \mathrm{H}$ NMR (DMSO, $400 \mathrm{MHz}$ )
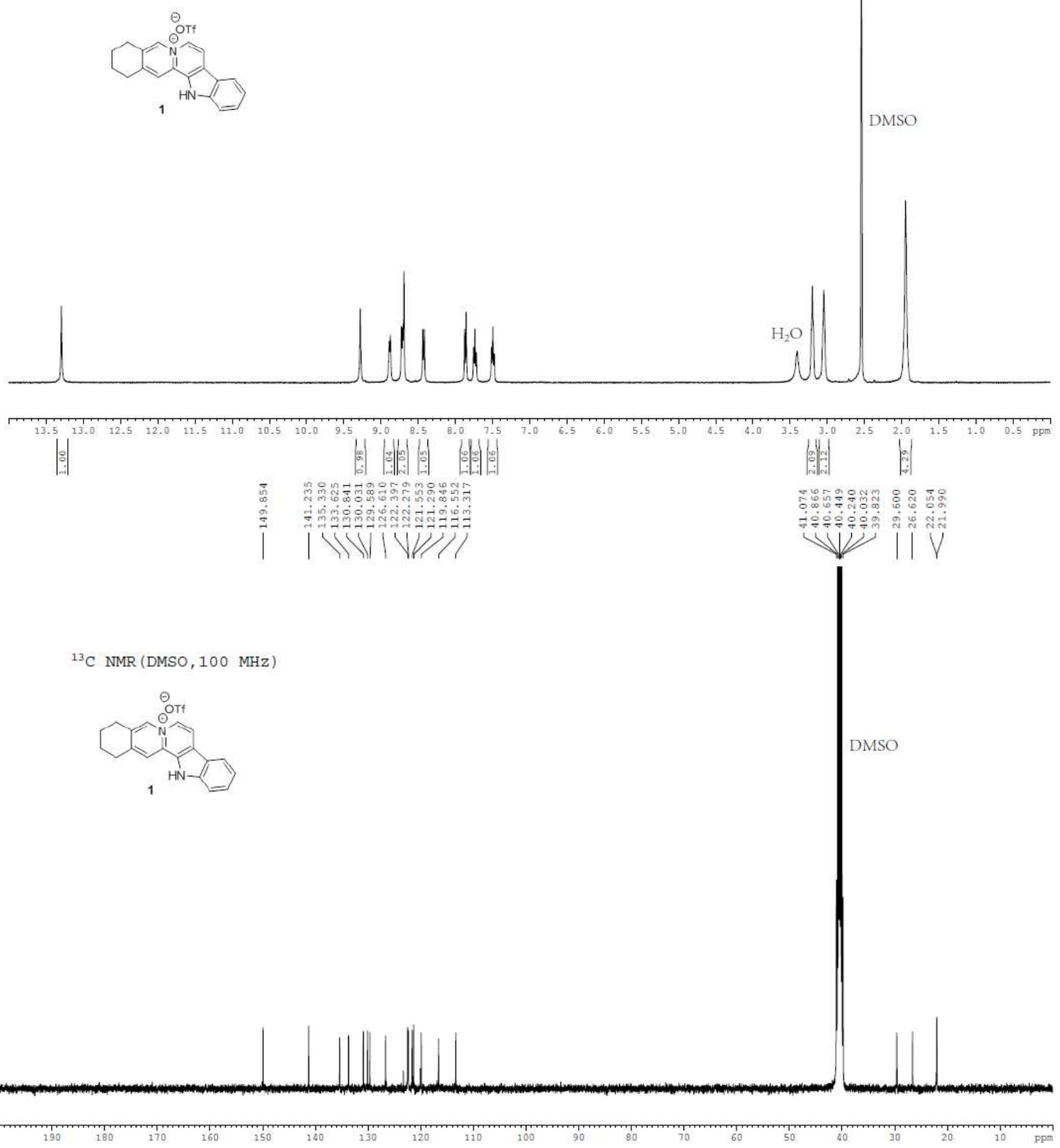
${ }^{1} \mathrm{H}$ and ${ }^{13} \mathrm{C}$ NMR spectrum of $3\left(\mathrm{CDCl}_{3}\right)$
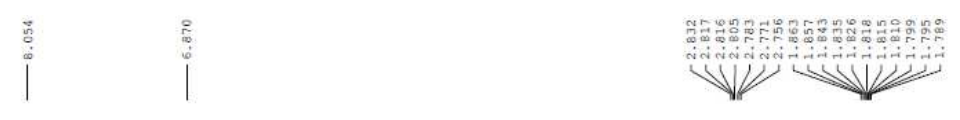

${ }^{1} \mathrm{H} \mathrm{NMR}\left(\mathrm{CDCl}_{3}, 400 \mathrm{MHz}\right)$

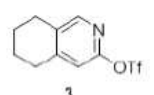

3

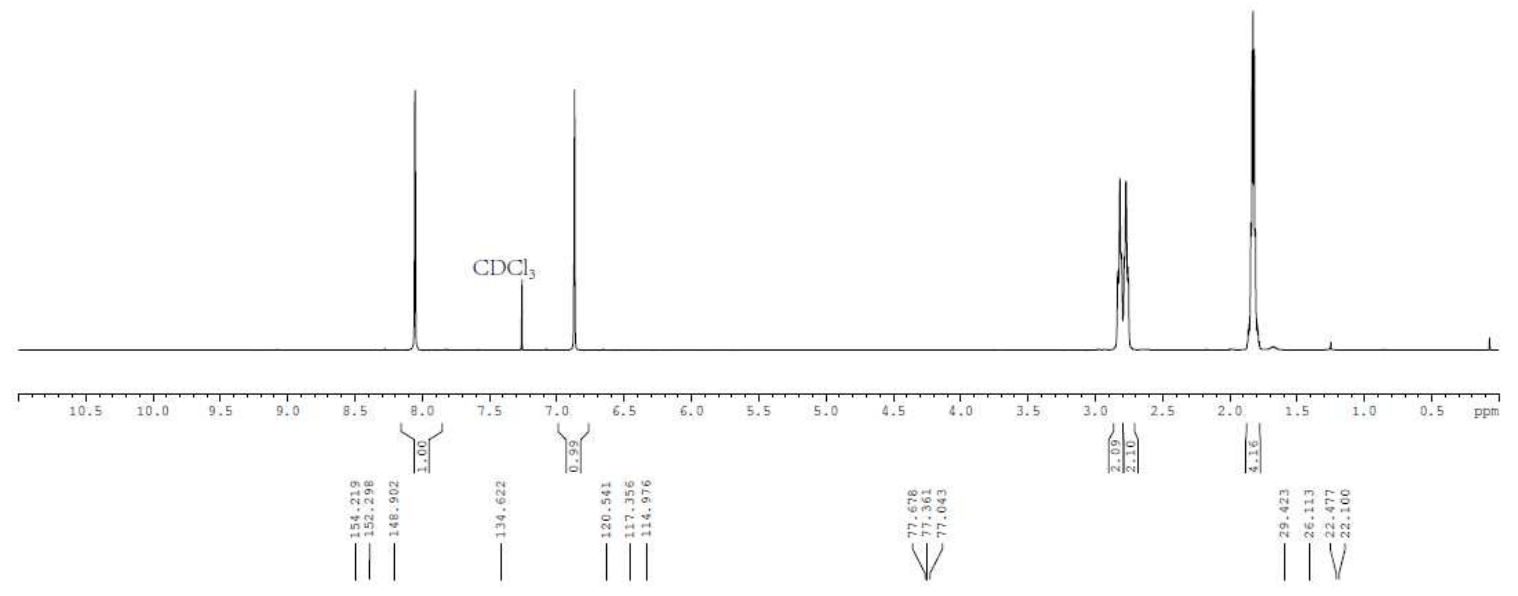

${ }^{13} \mathrm{C} \operatorname{NMR}\left(\mathrm{CDCl}_{3}, 100 \mathrm{MHz}\right)$
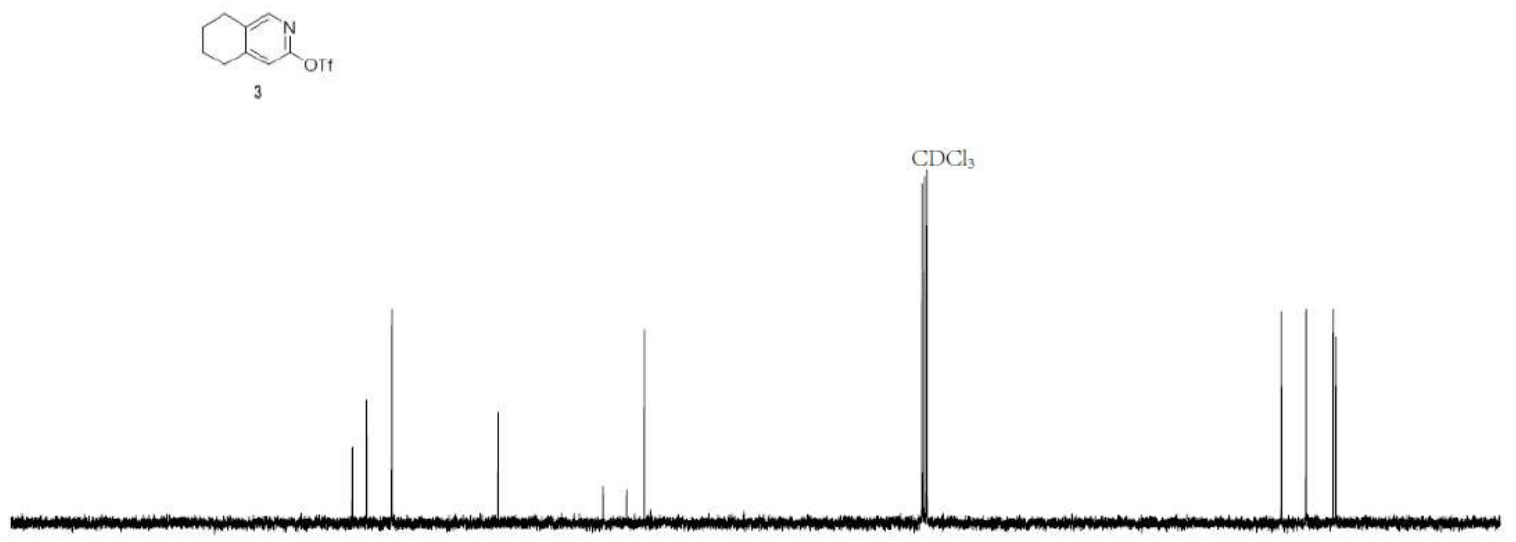


\section{${ }^{1} \mathrm{H}$ and ${ }^{13} \mathrm{C}$ NMR spectrum of $5\left(\mathrm{CDCl}_{3}\right)$}

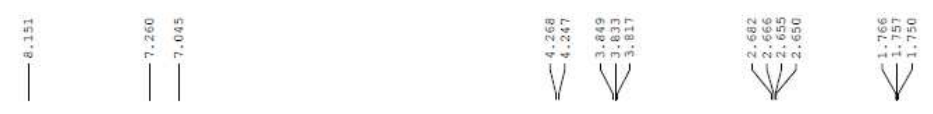

${ }^{1} \mathrm{H} \operatorname{NMR}\left(\mathrm{CDCl}_{3}, 400 \mathrm{MHz}\right)$
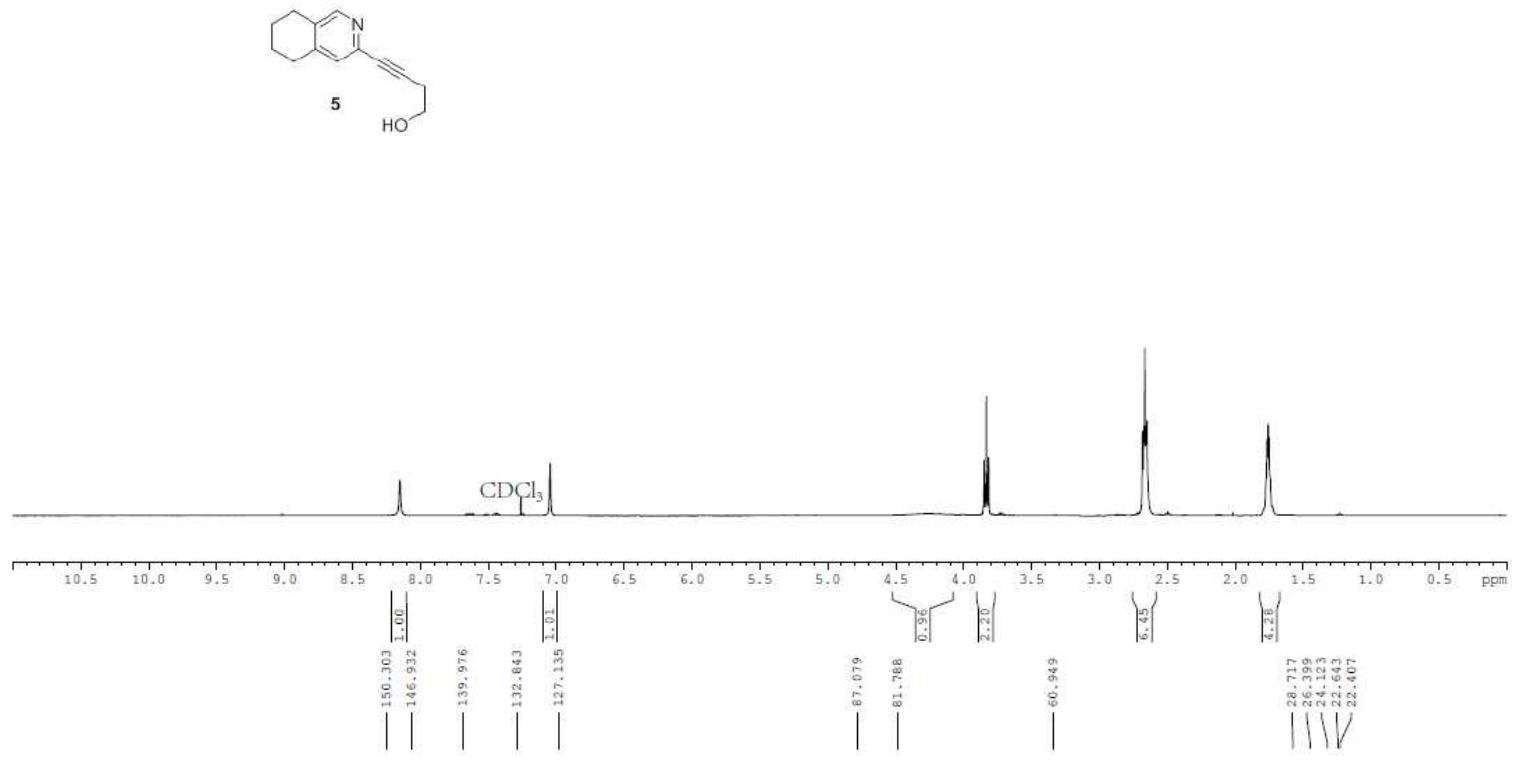

${ }^{13} \mathrm{C} \operatorname{NMR}\left(\mathrm{CDCl}_{3}, 100 \mathrm{MHz}\right)$

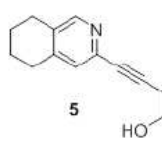

$\mathrm{CDCl}_{3}$

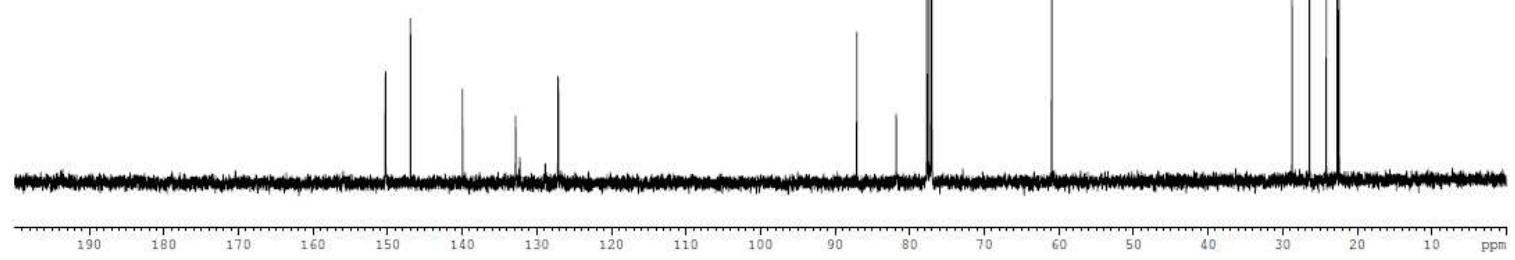


${ }^{1} \mathrm{H}$ and ${ }^{13} \mathrm{C}$ NMR spectrum of $7\left(\mathrm{CDCl}_{3}\right)$

|

${ }^{1} \mathrm{HNMR}\left(\mathrm{CDCl}_{3}, 400 \mathrm{MHz}\right)$
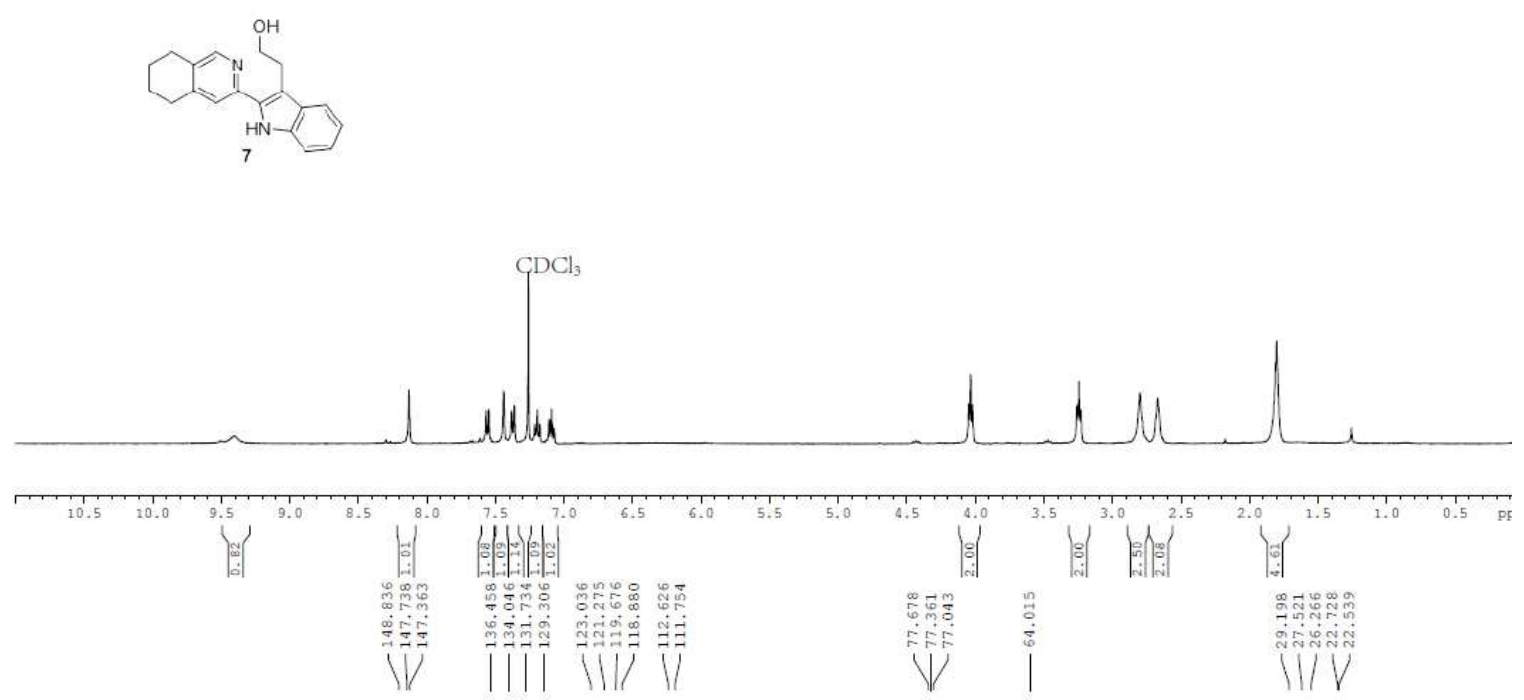

${ }^{13} \mathrm{CNMR}\left(\mathrm{CDCl}_{3}, 100 \mathrm{MHz}\right)$

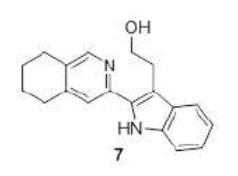

$\mathrm{CDCl}_{3}$

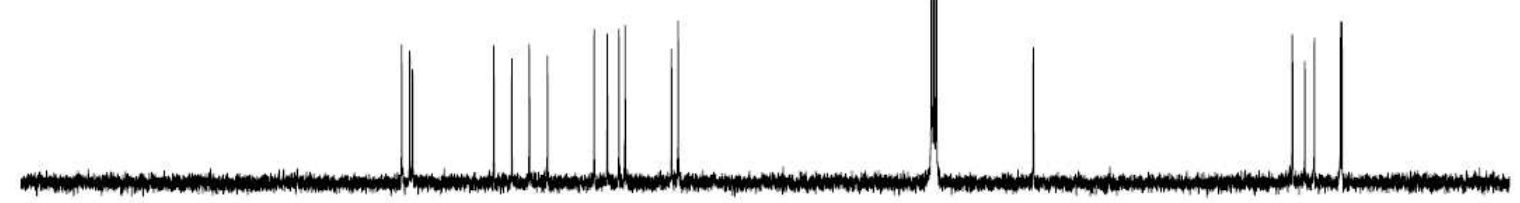




\section{${ }^{1} \mathrm{H}$ and ${ }^{13} \mathrm{C}$ NMR spectrum of 8 (DMSO)}

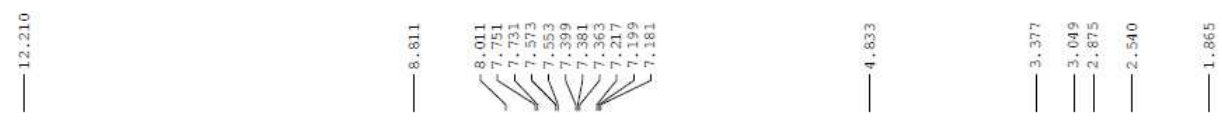

${ }^{1} \mathrm{H}$ NMR (DMSO, $400 \mathrm{MHz}$ )

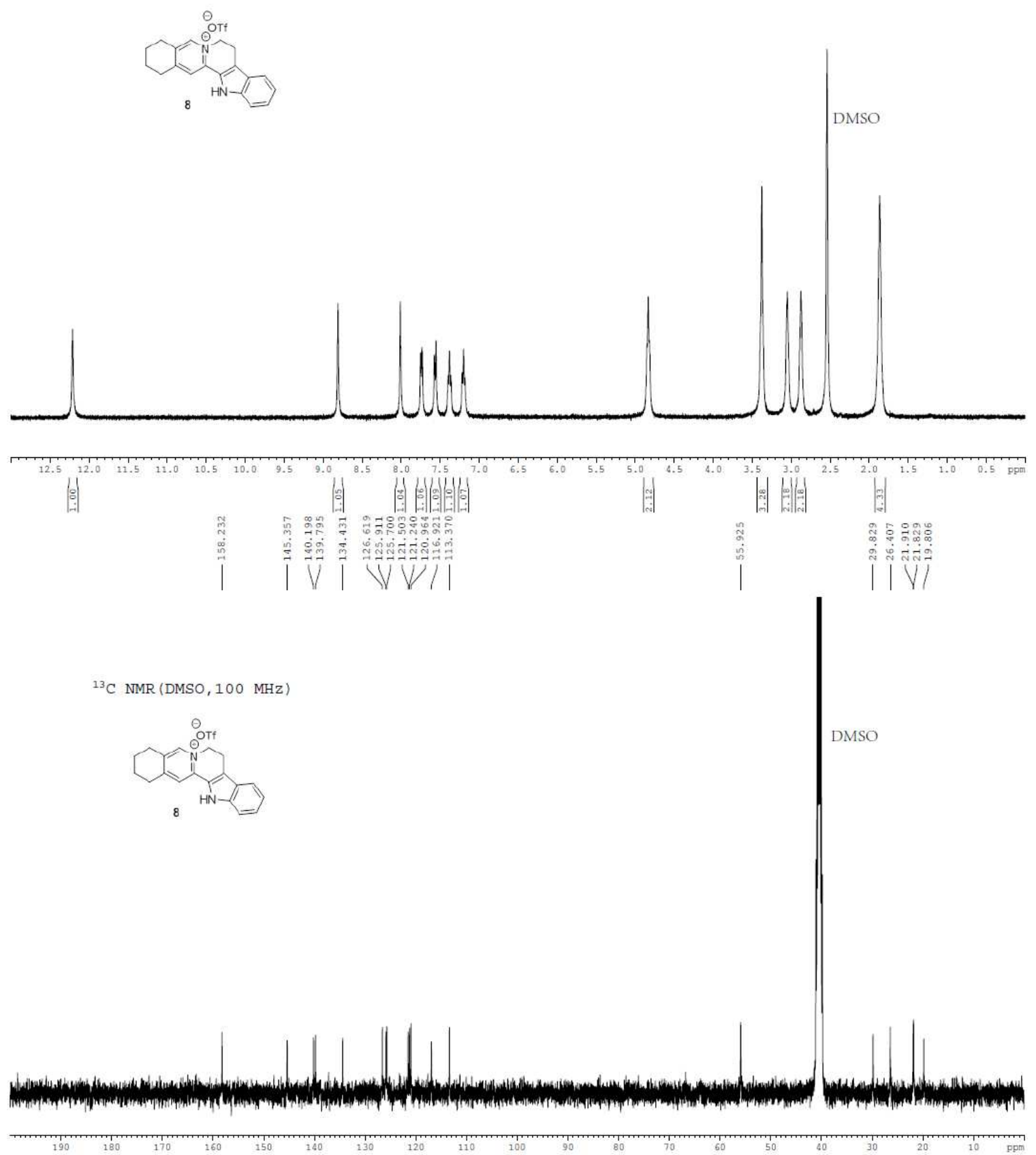




\section{${ }^{1} \mathrm{H}$ and ${ }^{13} \mathrm{C}$ NMR spectrum of $10\left(\mathrm{CDCl}_{3}\right)$}

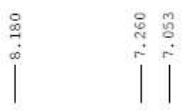

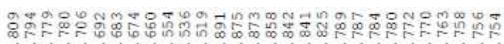

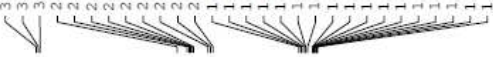

${ }^{1} \mathrm{H} \mathrm{NMR}\left(\mathrm{CDCl}_{3}, 400 \mathrm{MHz}\right)$
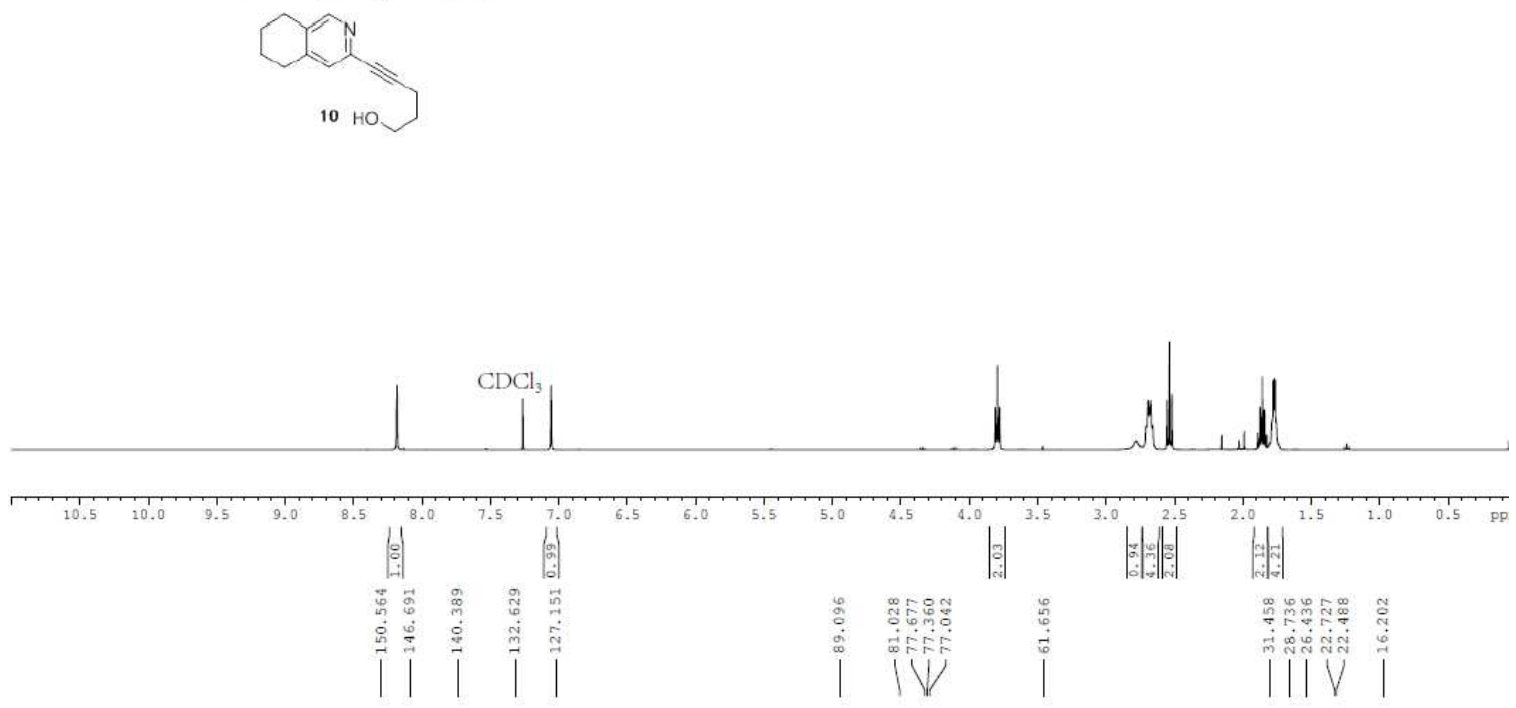

${ }^{13} \mathrm{C} \mathrm{NMR}\left(\mathrm{CDCl}_{3}, 100 \mathrm{MHz}\right)$

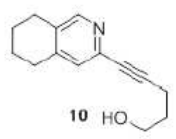

$\mathrm{CDCl}_{3}$

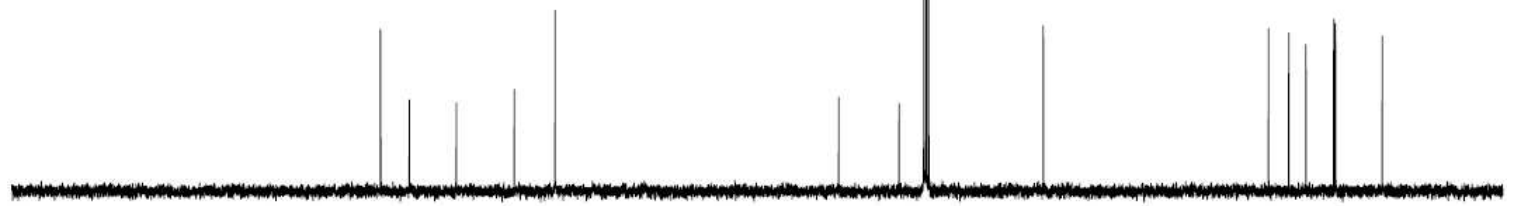


${ }^{1} \mathrm{H}$ and ${ }^{13} \mathrm{C}$ NMR spectrum of $11\left(\mathrm{CDCl}_{3}\right)$

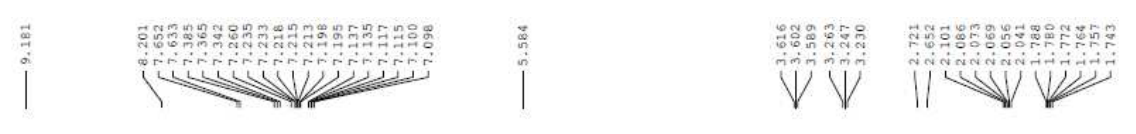

${ }^{1} \mathrm{H} \mathrm{NMR}\left(\mathrm{CDCl}_{3}, 400 \mathrm{MHz}\right)$
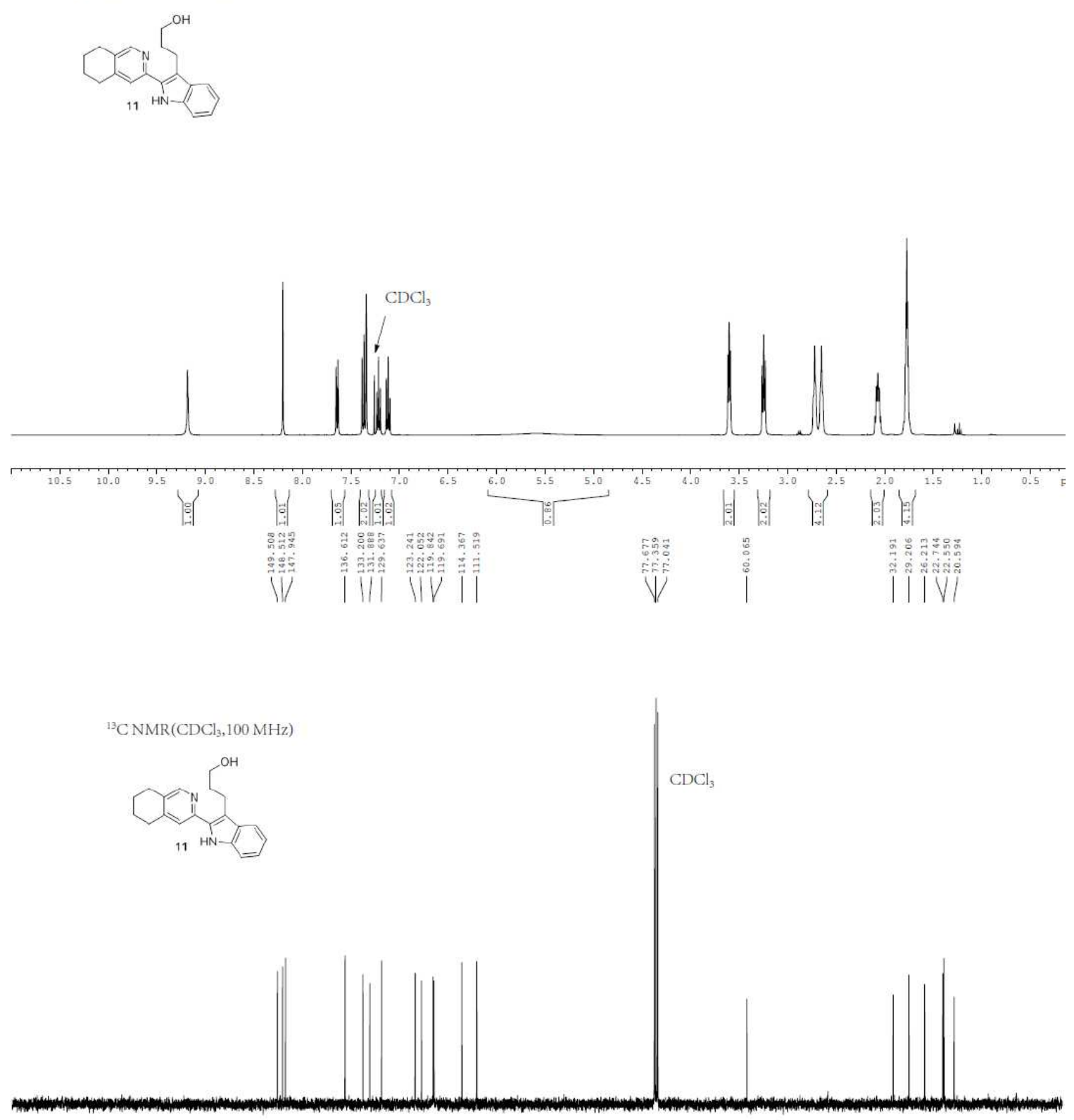


\section{${ }^{1} \mathrm{H}$ and ${ }^{13} \mathrm{C}$ NMR spectrum of 12 (DMSO)}

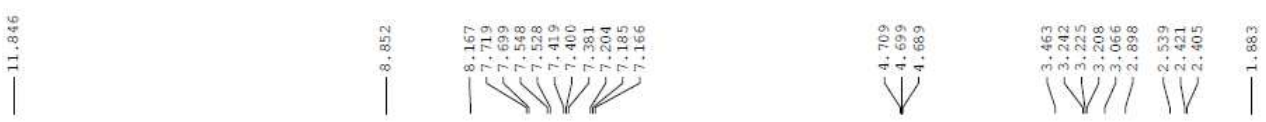

${ }^{\mathrm{I}} \mathrm{H} \mathrm{NMR}$ (DMSO, $400 \mathrm{MHz}$ )
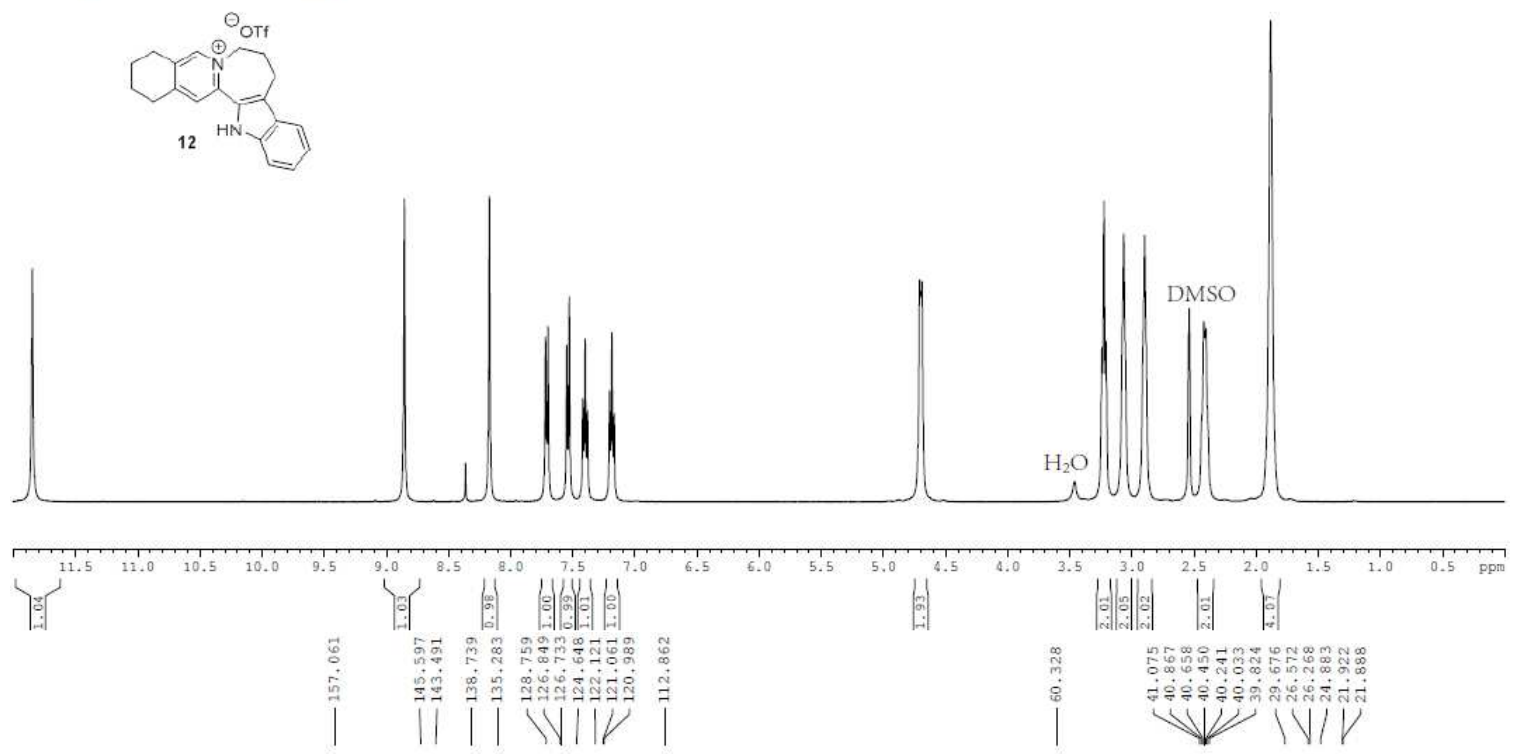

${ }^{13} \mathrm{C}$ NMR (DMSO, $\left.100 \mathrm{MHz}\right)$

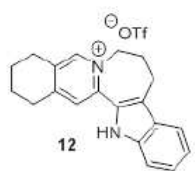

DMSO

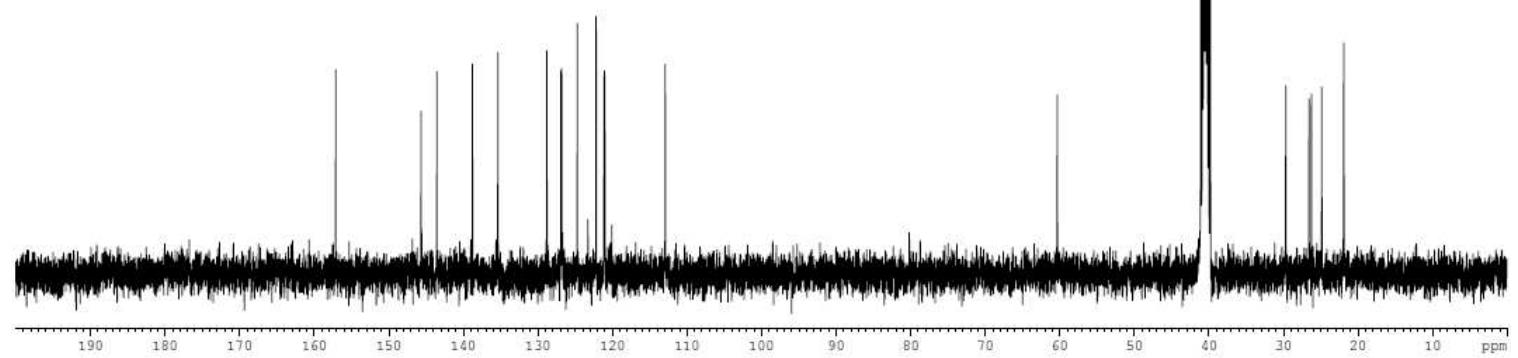




\section{${ }^{1} \mathrm{H}$ and ${ }^{13} \mathrm{C}$ NMR spectrum of $14\left(\mathrm{CDCl}_{3}\right)$}

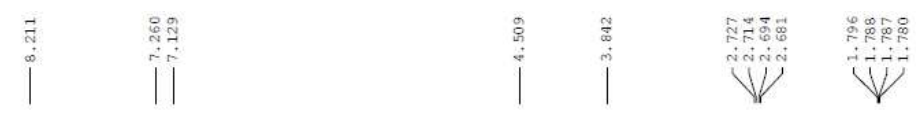

${ }^{1} \mathrm{H} \mathrm{NMR}\left(\mathrm{CDCl}_{3}, 400 \mathrm{MHz}\right)$
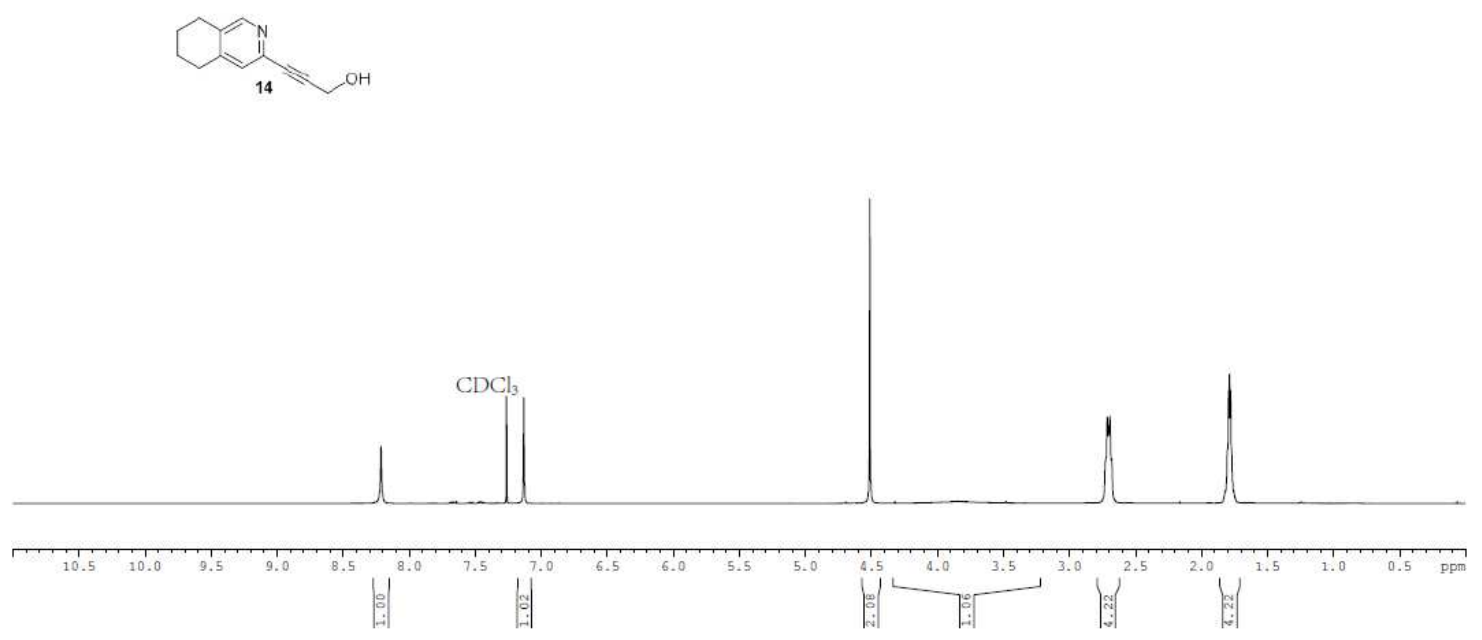

|

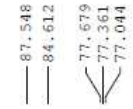

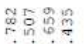

II V

${ }^{13} \mathrm{C} \mathrm{NMR}\left(\mathrm{CDCl}_{3}, 100 \mathrm{MHz}\right)$

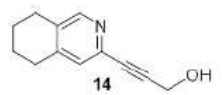




\section{${ }^{1} \mathrm{H}$ and ${ }^{13} \mathrm{C}$ NMR spectrum of $18\left(\mathrm{CDCl}_{3}\right)$}
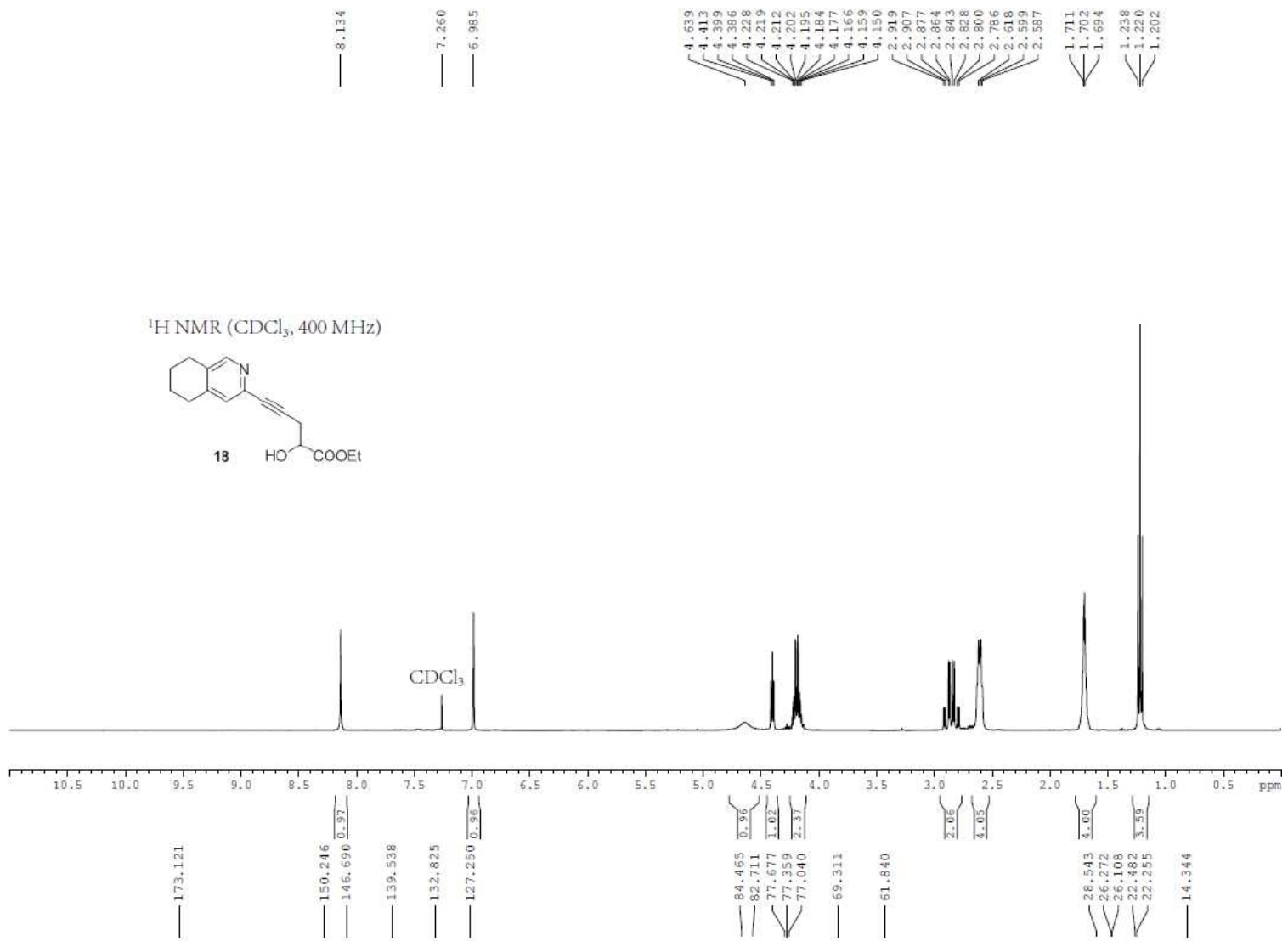

${ }^{13} \mathrm{C}$ NMR $\left(\mathrm{CDCl}_{3}, 100 \mathrm{MHz}\right)$
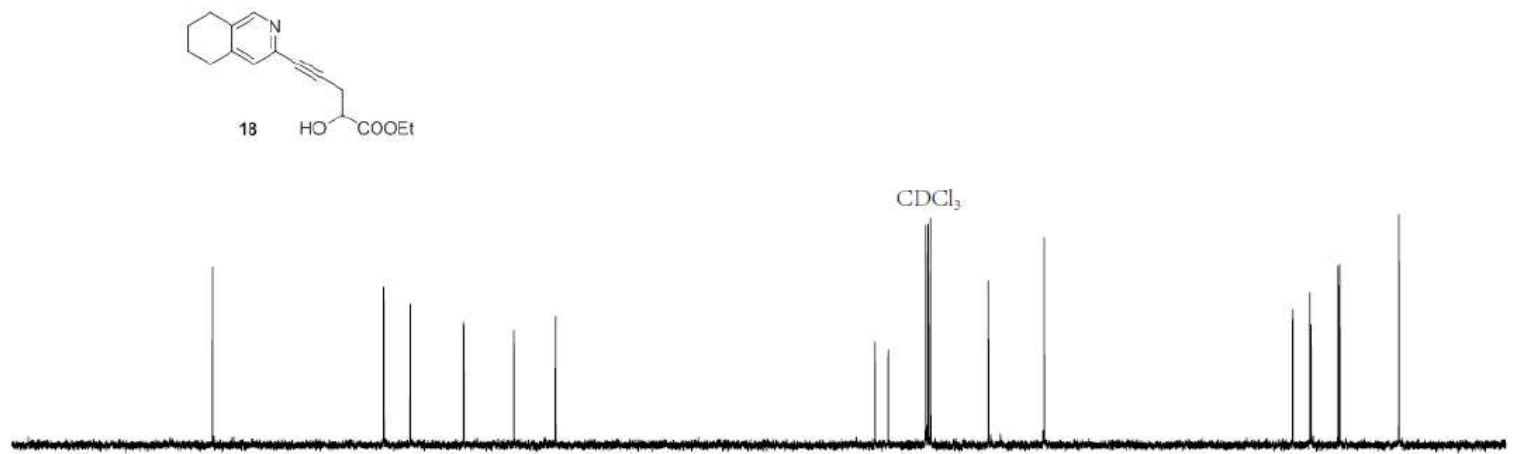


\section{${ }^{1} \mathrm{H}$ and ${ }^{13} \mathrm{C}$ NMR spectrum of $19\left(\mathrm{CDCl}_{3}\right)$}

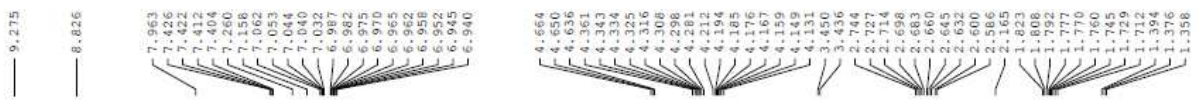

${ }^{1} \mathrm{H} \mathrm{NMR}\left(\mathrm{CDCl}_{3}, 400 \mathrm{MHz}\right)$
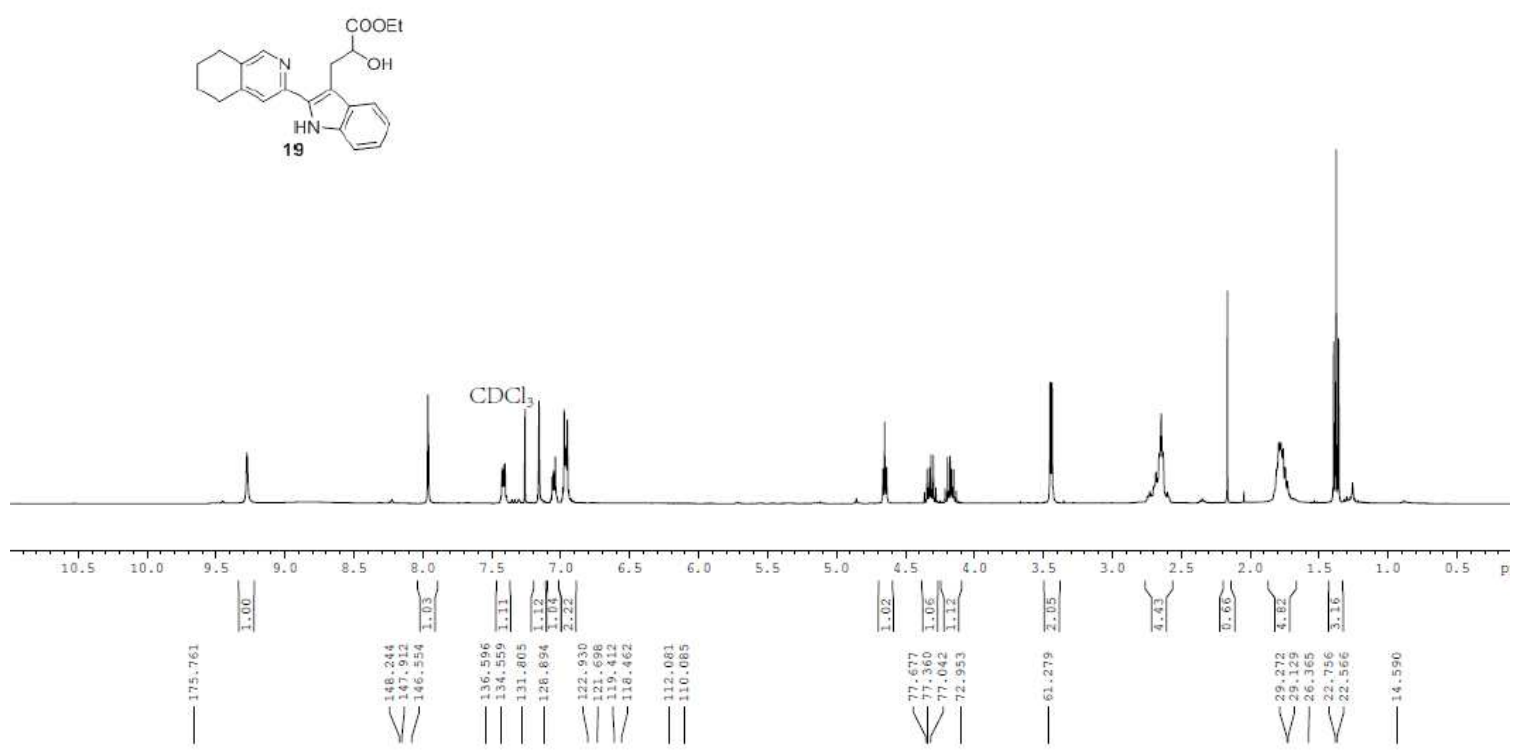

${ }^{13} \mathrm{C} \mathrm{NMR}\left(\mathrm{CDCl}_{3}, 100 \mathrm{MHz}\right)$
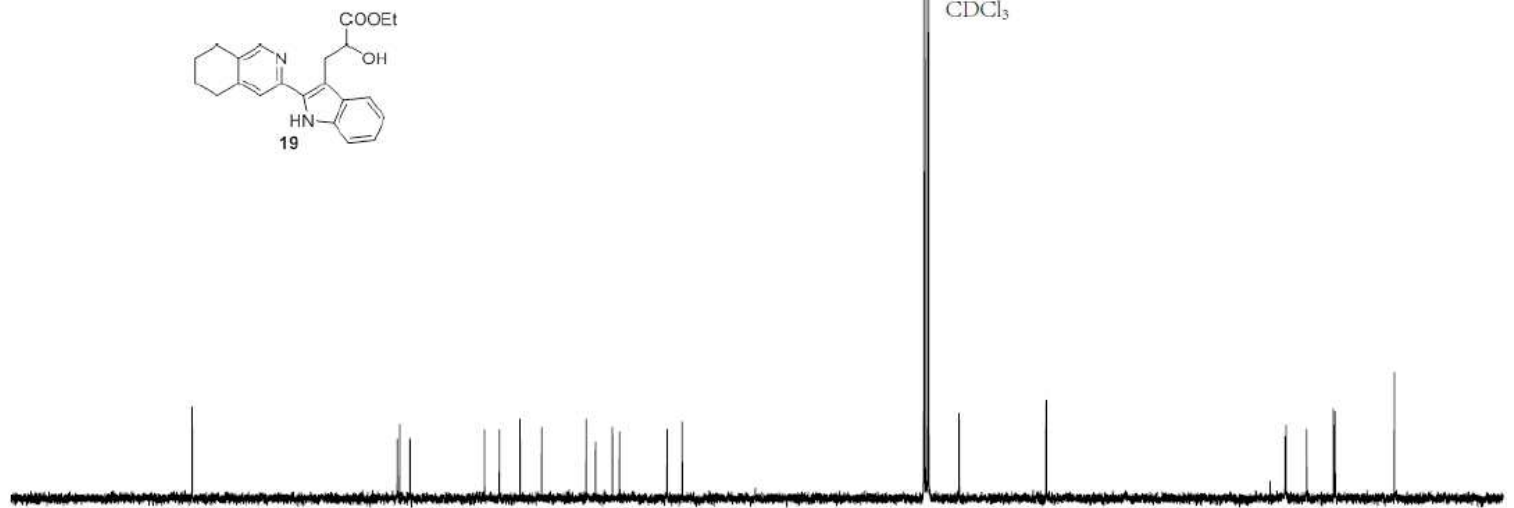


\section{${ }^{1} \mathrm{H}$ and ${ }^{13} \mathrm{C}$ NMR spectrum of 20 (DMSO)}

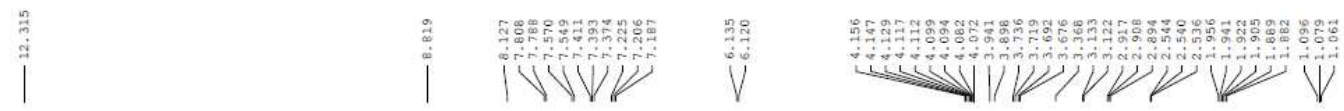

'H NMR (DMSO, $400 \mathrm{MHz}$ )
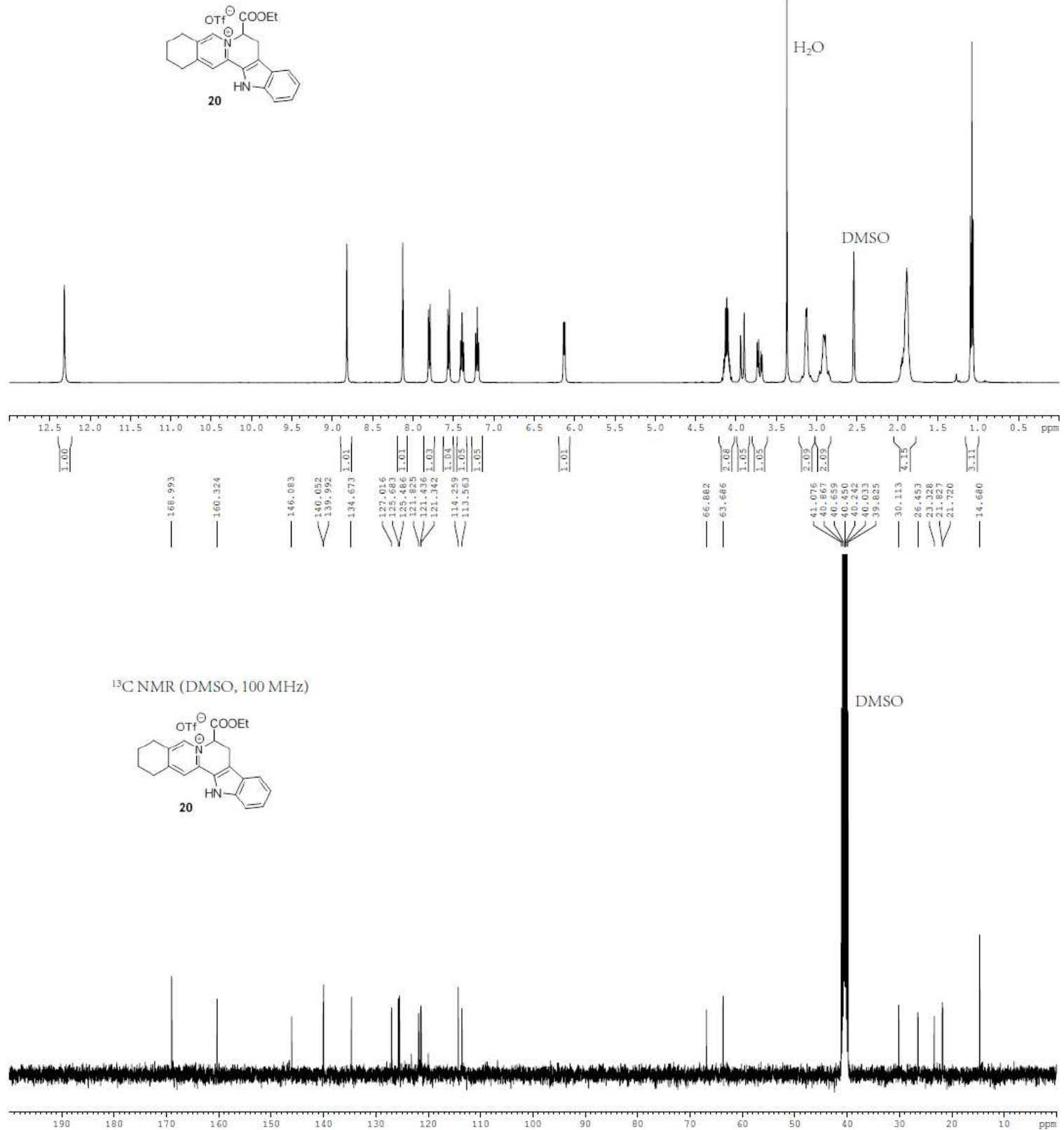


\section{${ }^{1} \mathrm{H}$ and ${ }^{13} \mathrm{C}$ NMR spectrum of 21 (DMSO)}

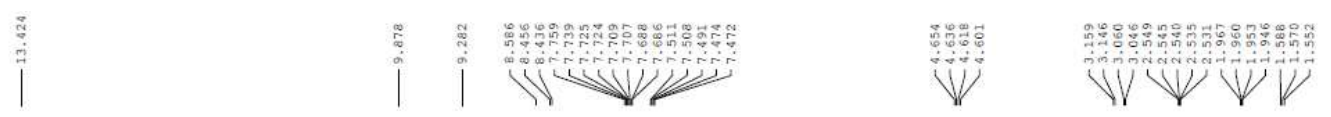

'H NMR (DMSO, $400 \mathrm{MHz}$ )
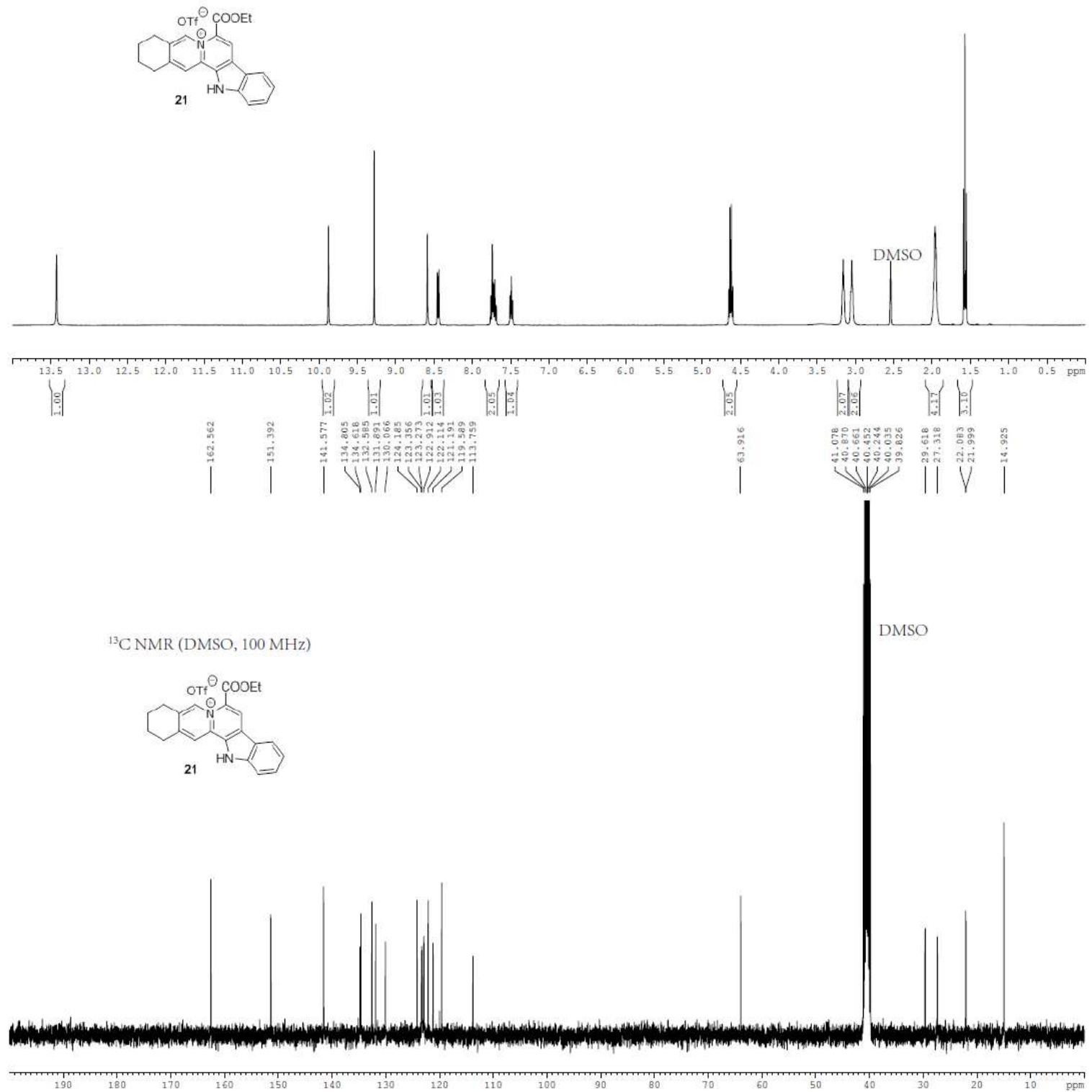


\section{${ }^{1} \mathrm{H}$ and ${ }^{13} \mathrm{C}$ NMR spectrum of 27 (DMSO)}
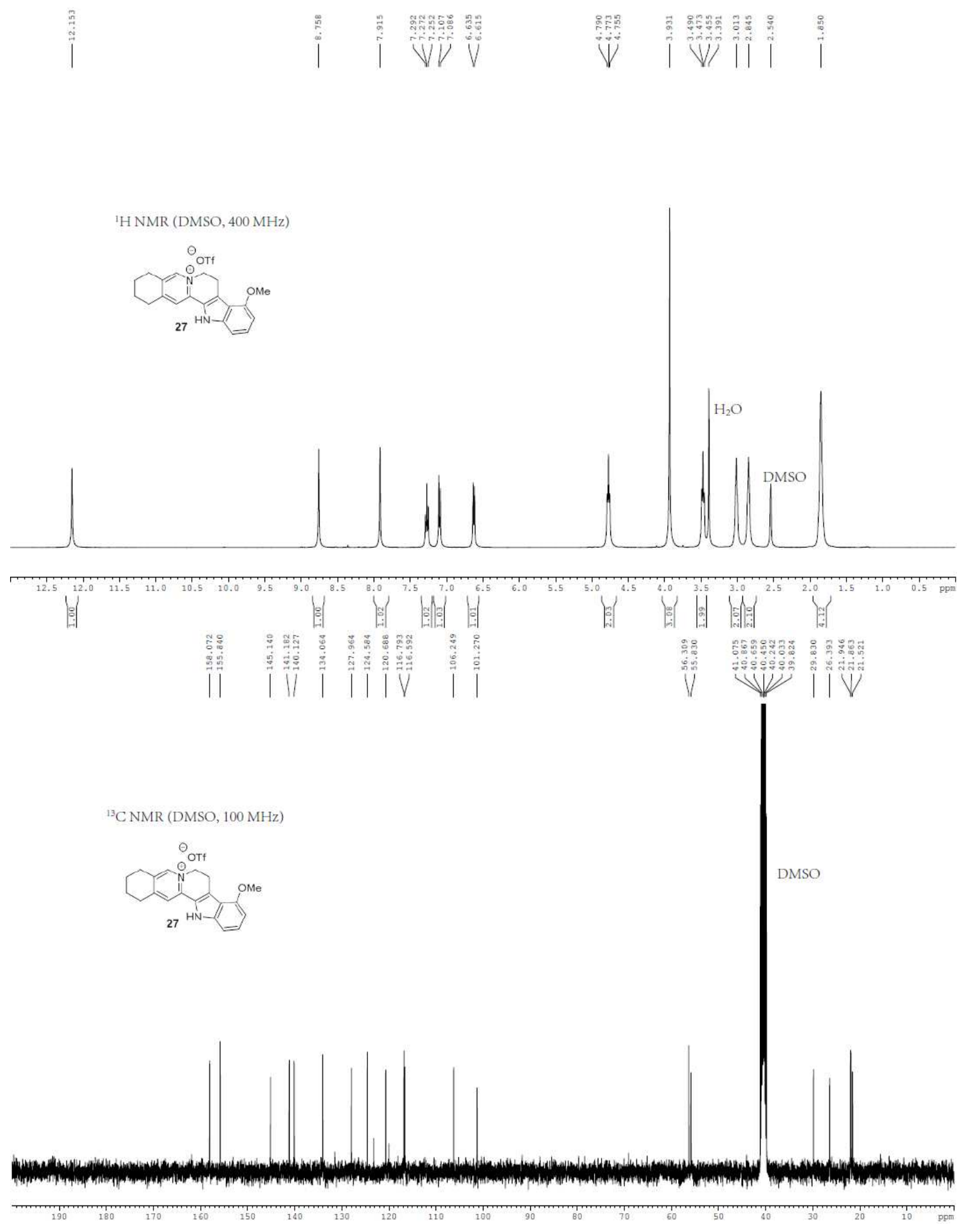


\section{${ }^{1} \mathrm{H}$ and ${ }^{13} \mathrm{C}$ NMR spectrum of 28 (DMSO)}

${ }^{1} \mathrm{H}$ NMR (DMSO, $400 \mathrm{MHz}$ )
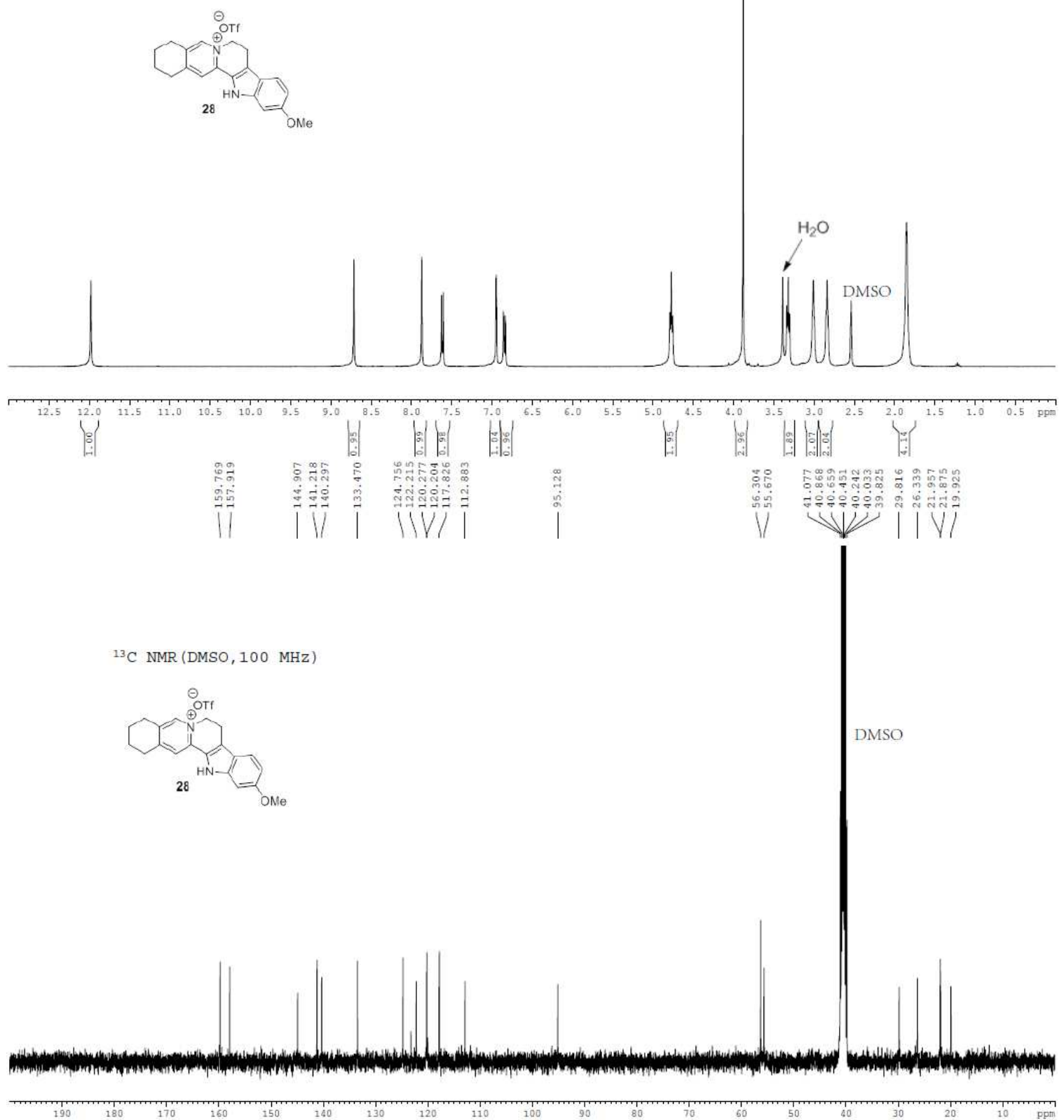


\section{${ }^{1} \mathrm{H}$ and ${ }^{13} \mathrm{C}$ NMR spectrum of 29 (DMSO)}
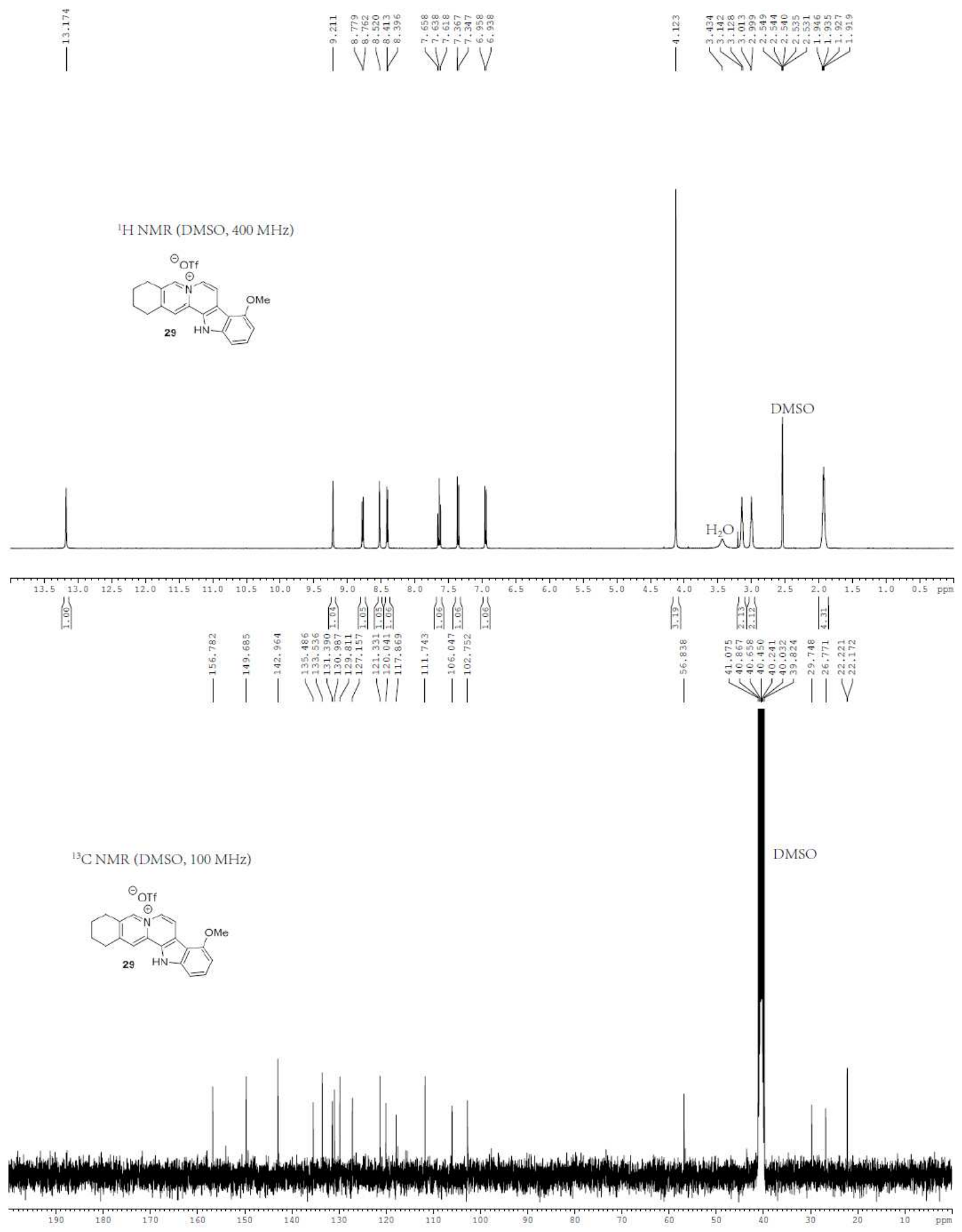


\section{${ }^{1} \mathrm{H}$ and ${ }^{13} \mathrm{C}$ NMR spectrum of 30 (DMSO)}

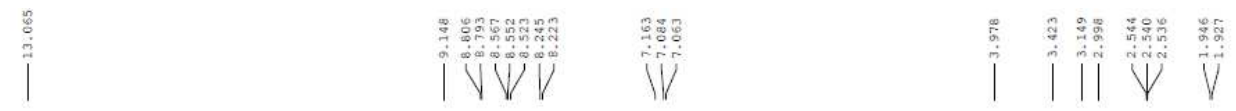

${ }^{1} \mathrm{H}$ NMR (DMSO, $400 \mathrm{MHz}$ )
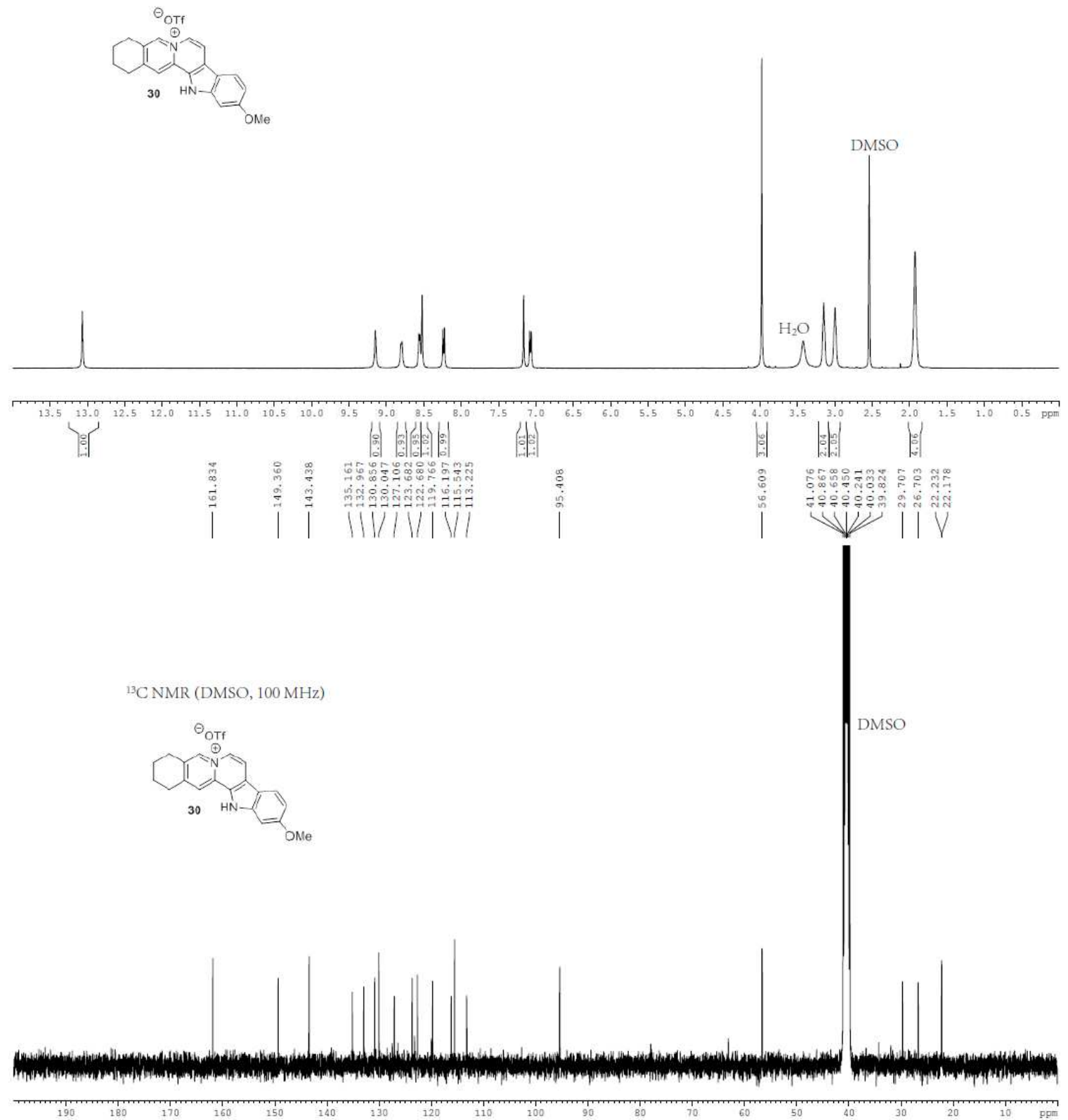


\section{${ }^{1} \mathrm{H}$ and ${ }^{13} \mathrm{C}$ NMR spectrum of 35 (DMSO)}

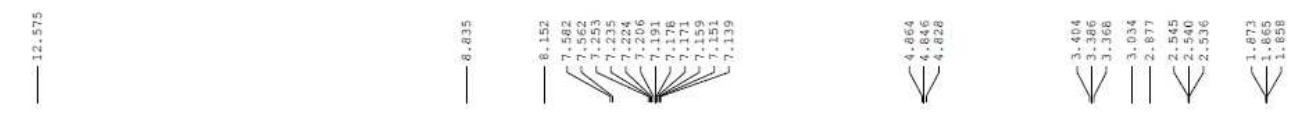

${ }^{1} \mathrm{H}$ NMR (DMSO, $400 \mathrm{MHz}$ )
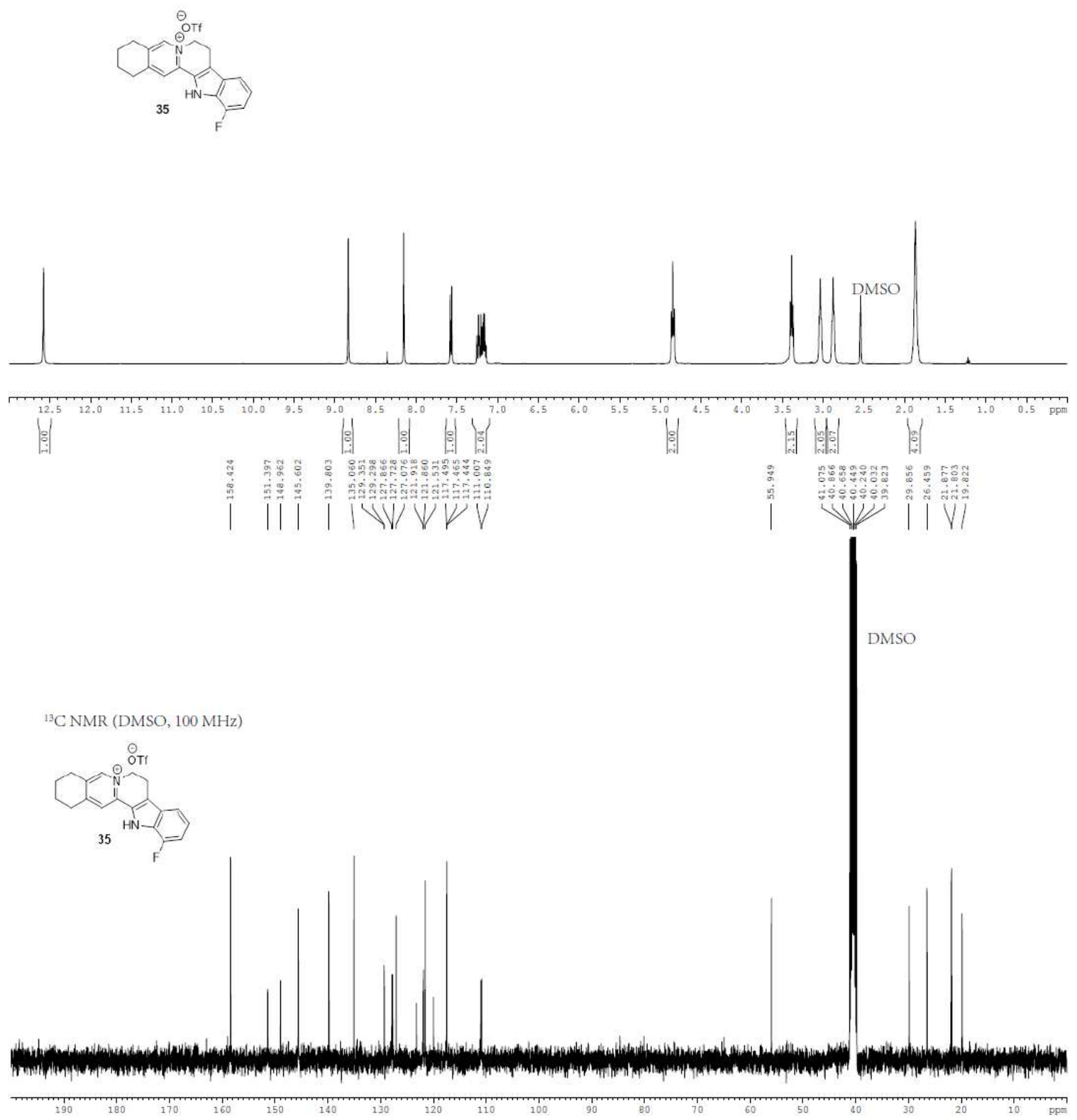
${ }^{1} \mathrm{H}$ and ${ }^{13} \mathrm{C}$ NMR spectrum of 36 (DMSO)

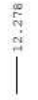
|
Vij
Vंj|| |

${ }^{1} \mathrm{H}$ NMR (DMSO, $400 \mathrm{MHz}$ )
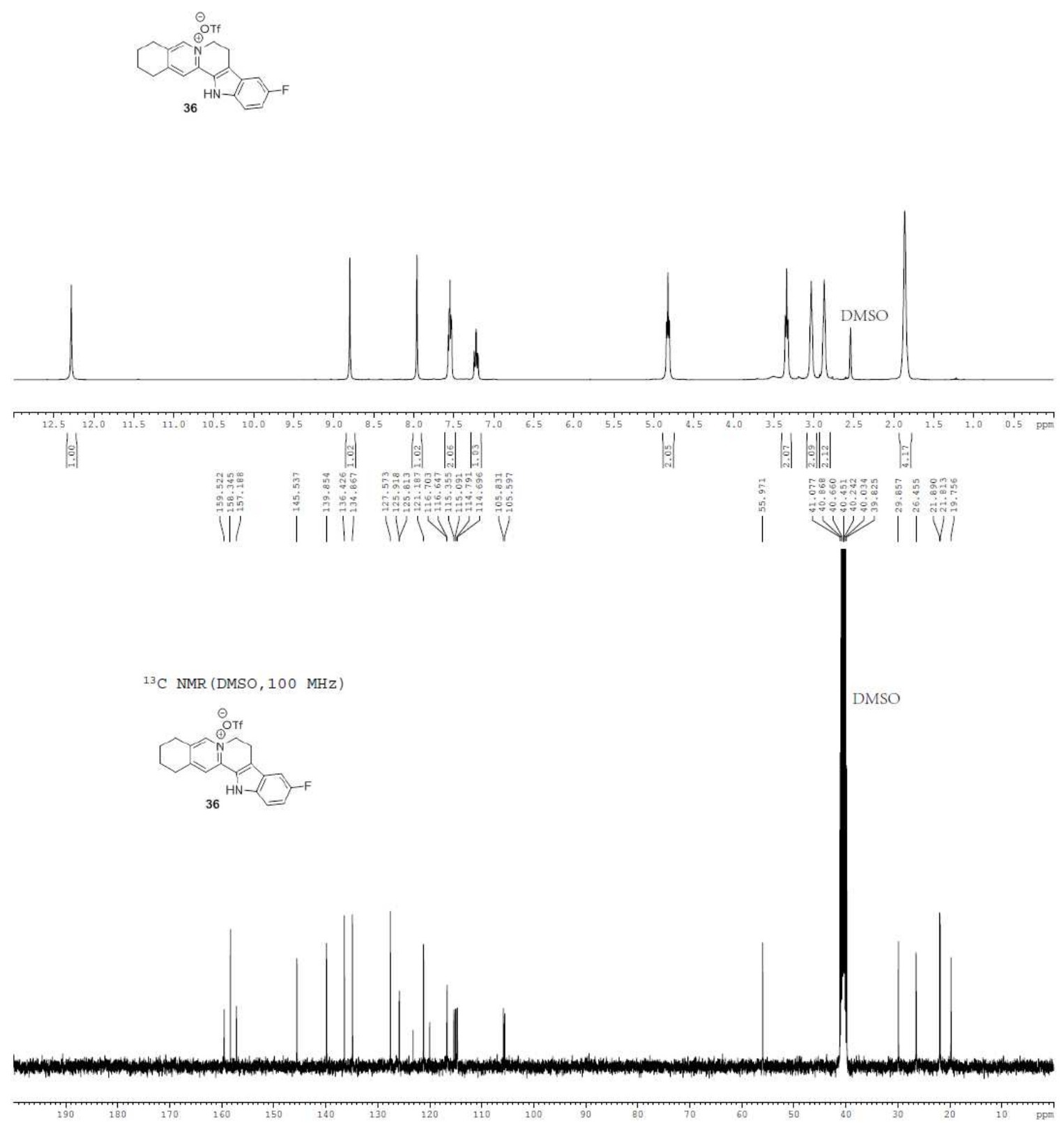


\section{${ }^{1} \mathrm{H}$ and ${ }^{13} \mathrm{C}$ NMR spectrum of 37 (DMSO)}

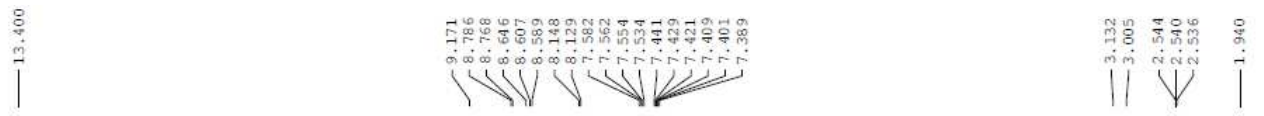

${ }^{1} \mathrm{H}$ NMR (DMSO, $400 \mathrm{MHz}$ )
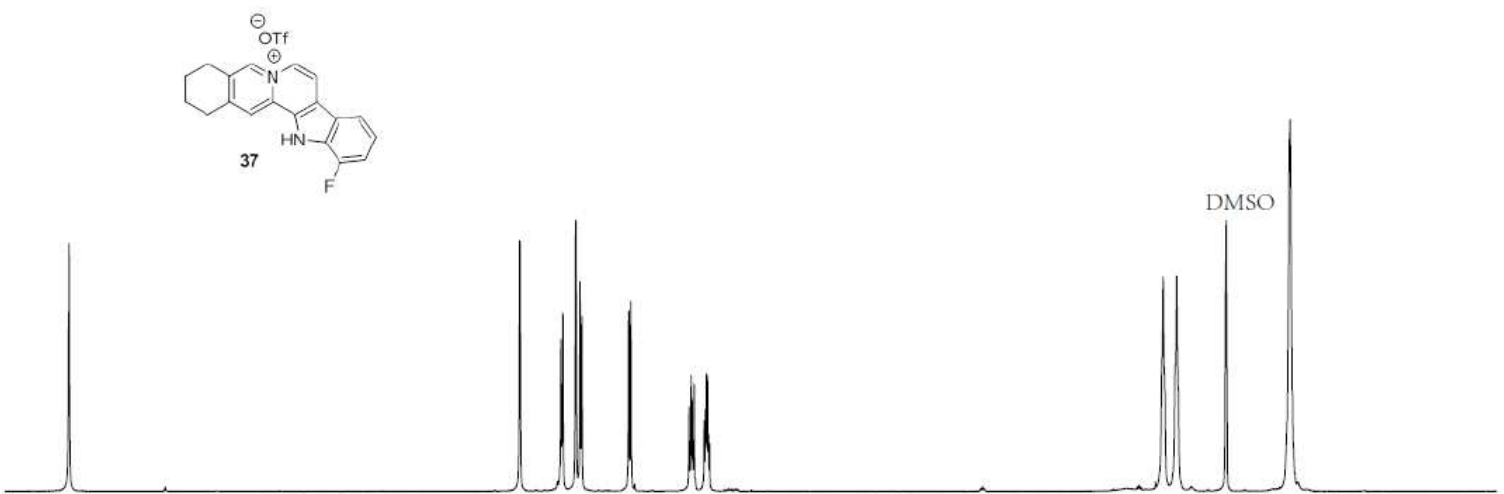

装

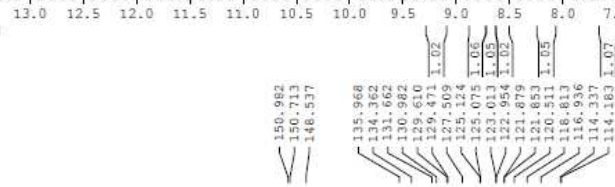

${ }^{13} \mathrm{C}$ NMR (DMSO, $100 \mathrm{MHz}$ )

DMSO

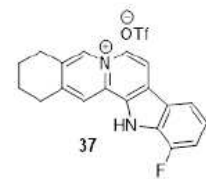

wint

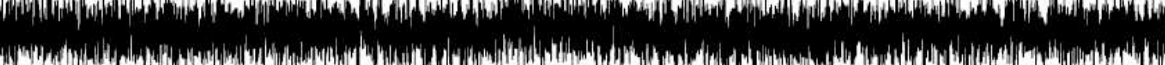

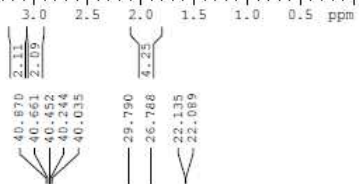




\section{${ }^{1} \mathrm{H}$ and ${ }^{13} \mathrm{C}$ NMR spectrum of 38 (DMSO)}
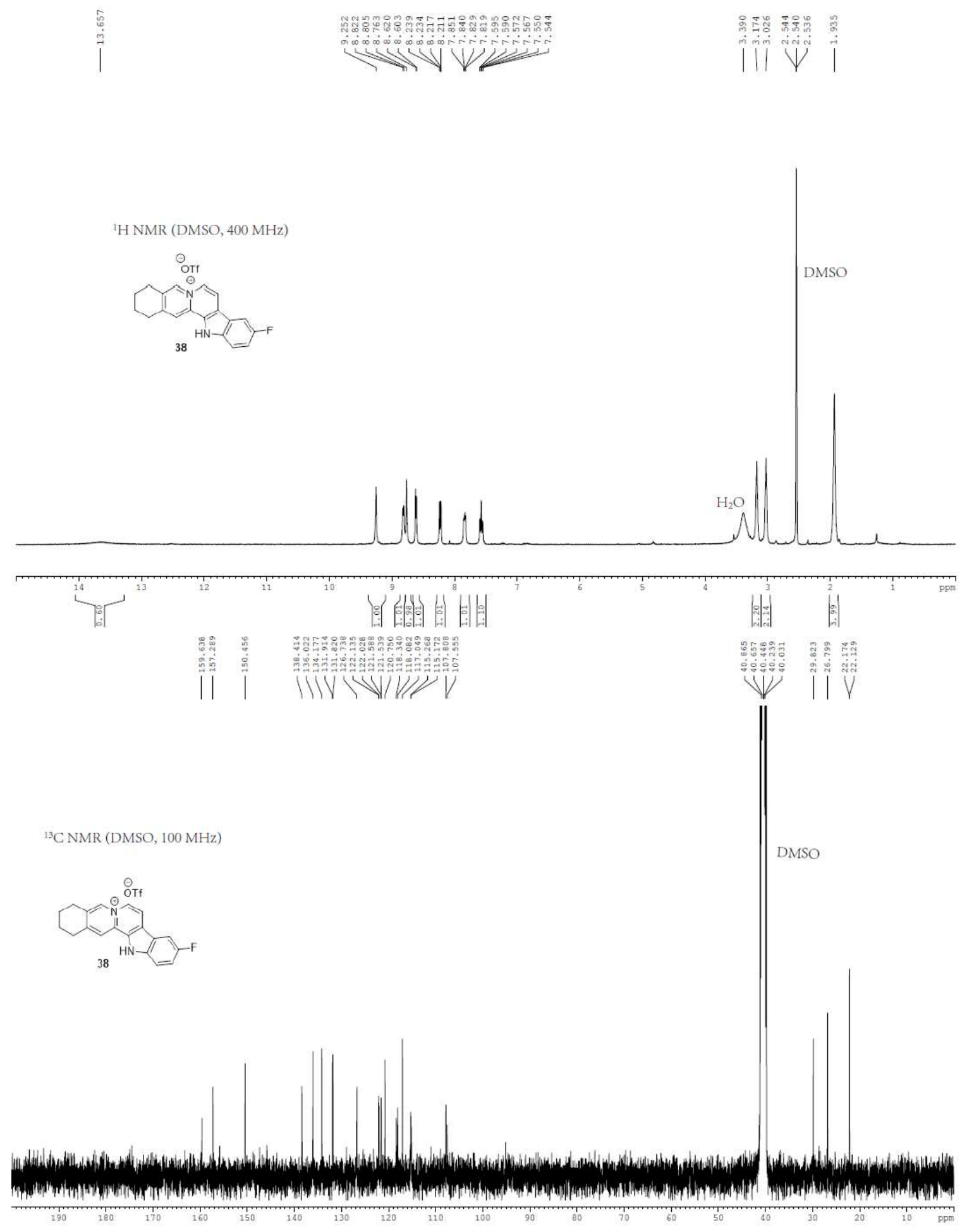


\section{${ }^{1} \mathrm{H}$ and ${ }^{13} \mathrm{C}$ NMR spectrum of 43 (DMSO)}

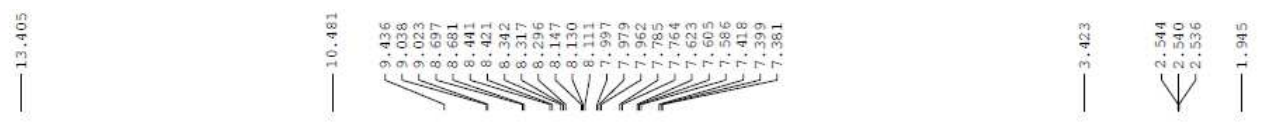

${ }^{1} \mathrm{H}$ NMR (DMSO, $400 \mathrm{MHz}$ )
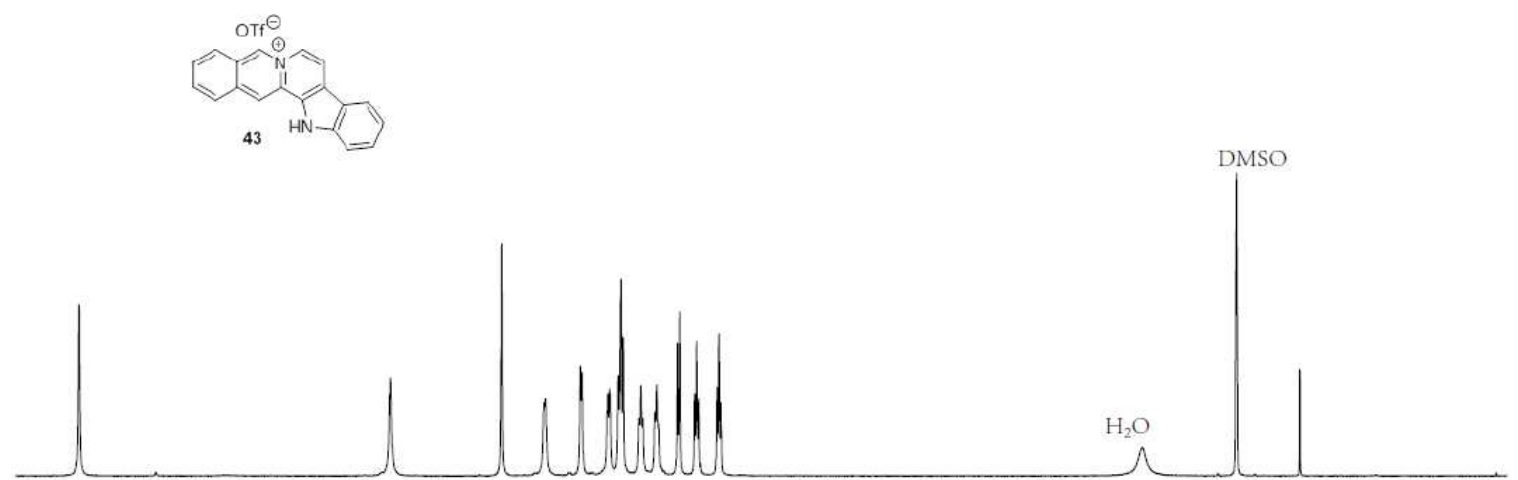

${ }^{13} \mathrm{C}$ NMR (DMSO, $100 \mathrm{MHz}$ )
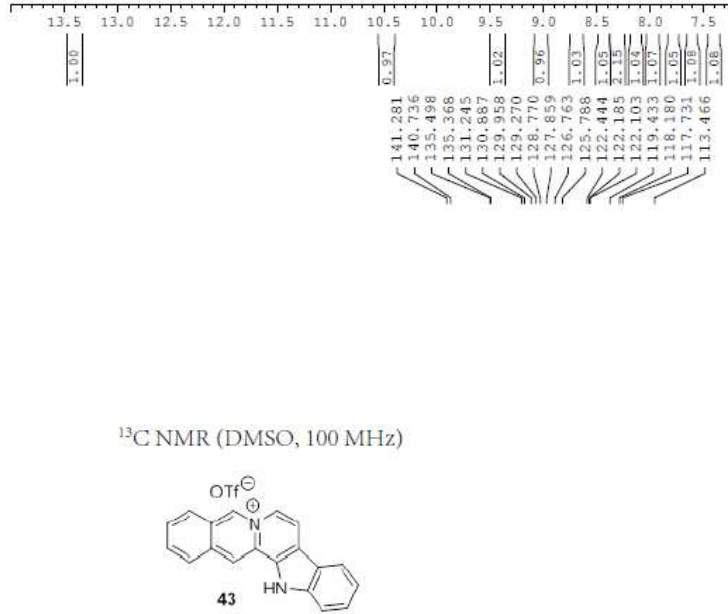

-600 in

-

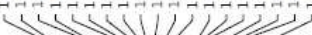
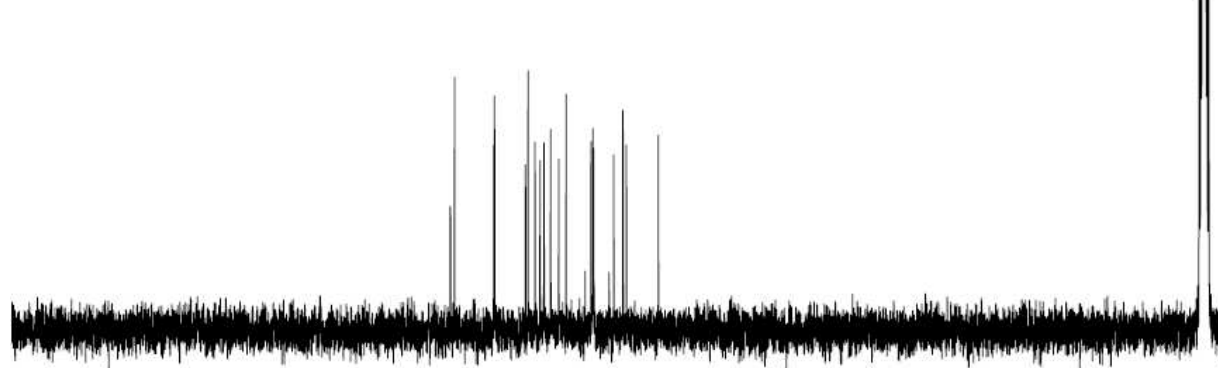

DMSO
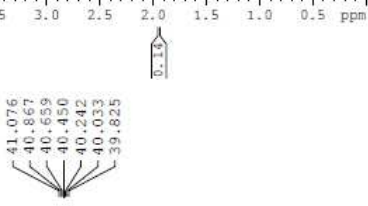

:

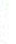

